\title{
IDENTIFICATION AND FUNCTIONAL CHARACTERIZATION OF TWO GRANULOCYTE COLONY-STIMULATING FACTORS OF COMMON CARP
}

\author{
Fumihiko Katakura $^{1 \S}$, Erika Hino ${ }^{1}$, Kohei Nishiya ${ }^{1}$, Jiro Miyamae ${ }^{1}$, Tadaaki Moritomo ${ }^{1}$ \\ ${ }^{I}$ Department of Veterinary Medicine, Nihon University, Fujisawa, Kanagawa, Japan
}

\begin{abstract}
Granulocyte colony-stimulating factor (GCSF) is a cytokine that regulates the proliferation, differentiation, survival and immunological activation of myeloid cells such as neutrophils and monocytes, and their progenitors in mammals. GCSF is used as a recombinant therapeutics against mammalian neutropenia to prevent infectious diseases. However, functions of GCSF are yet to be deeply characterized in lower vertebrates. Analysis of carp genome showed that carp have two paralogous GCSF molecules, GCSFa and GCSFb, similar to other teleosts, presumably resulting from a whole genome duplication event in teleosts. These molecules were predicted to possess functional domains and structures, although there are quite low sequence identities between vertebrate species. Expression analysis of carp gcsfa and gcsfb revealed the highest gcsfa mRNA levels in spleen, while the highest gcsfb mRNA levels in heart. Both GCSFa and GCSFb appear to be involved in immunity based on their up-regulation in kidney leukocytes following in vitro stimulation of lipopolysaccharide and a combination of Concanavalin A and Phorbol myristate acetate. Additionally, recombinant carp GCSFa and GCSFb stimulated the proliferation and the colony-formation of carp kidney leukocytes in a dose dependent manner, suggesting that both ligands play a role as a hematopoietic growth factor. Furthermore, recombinant GCSFb induced the development of morphologically mature neutrophils and up-regulated mRNA expression of GCSF receptor in the kidney leukocytes. Our results indicate that carp GCSFs are involved in the immune system and at least GCSFb has parallel functions to mammalian GCSF as a regulator of neutrophilic myelopoiesis.
\end{abstract}

\section{KEYWORDS}

Granulocyte colony-stimulating factor; Common carp; Hematopoiesis; Neutrophils; In vitro

${ }^{\S}$ Corresponding author. Tel.: +81 (0)466 84 3443; Fax: +81 (0)466 843374.

E-mail address: katakura.fumihiko@nihon-u.ac.jp 